\title{
PHOTOMETRIC STUDY OF THREE SHORT PERIOD VARIABLE STARS ${ }^{1}$
}

\author{
J. H. Peña ${ }^{2,3,4}$, S. B. Juárez ${ }^{4}$, A. A. Soni ${ }^{2,4}$, H. Huepa ${ }^{3}$, A. Rentería ${ }^{2,3,4}$, and A. L. Zuñiga ${ }^{4}$ \\ Received November 26 2019; accepted July 292020
}

\begin{abstract}
We have obtained $u v b y-\beta$ photoelectric photometry with the $0.84 \mathrm{~m}$ telescope at the San Pedro Mártir Observatory, México, for the stars HD 115520, HD 220735 and HD 26738. The first two had been previously determined to be variables whereas the last one is being reported here as a new variable. With an extended time basis for the first two, we have determined their period of pulsation, which is found to be stable. Physical parameters are presented for the three stars.

\section{RESUMEN}

Se obtuvo fotometría fotoeléctrica uvby - $\beta$ con el telescopio de $0.84 \mathrm{~m}$ del Observatorio de San Pedro Mártir, México, de las estrellas HD115520, HD 220735 y HD 26738; las primeras dos ya se habían determinado como variables mientras que la última se reporta aquí por primera vez como variable. Con la base de tiempo ampliada de las dos primeras determinamos sus períodos de pulsación, que se encuentran estables. Se presentan parámetros físicos para las tres estrellas.
\end{abstract}

Key Words: stars: variables: delta Scuti — stars: variables: general — techniques: photometric

\section{INTRODUCTION}

Knowledge of the nature of variable stars can be achieved with only a few hours of observation, but a more profound understanding can be attained solely through continuous monitoring. For this reason it is important for them to be observed and reported. A previous study (Fox Machado et al. 2007) confirmed the variable nature of HD 115520 as a $\delta$ Scuti star, a state which had been previously determined by Peña et al. (2007). In this paper we present unpublished observations of this star which were carried out in 2013; these data permitted the analysis of the periodic content with an extended time baseline. Analogously, HD 220735 was found to be variable, and was reported in Peña et al. (2019) with observational data from 2016. A new set of observations over two nights in 2018 is presented in the current paper. The new period analysis establishes that, as in the case of HD 115520, two close frequencies pro-

\footnotetext{
${ }^{1}$ Based on observations collected at the San Pedro Mártir Observatory, México.

${ }^{2}$ Instituto de Astronomía, Universidad Nacional Autónoma de México, México.

${ }^{3}$ Observatorio Astronómico Nacional de Tonantzintla, México.

${ }^{4}$ Facultad de Ciencias, Universidad Nacional Autónoma de México, México.
}

voke modulation in its light curve. HD 26738 was found to be a new, previously unreported, variable star.

\section{OBSERVATIONS}

These were all done at the Observatorio Astronómico Nacional de San Pedro Mártir, México (SPM). The $0.84 \mathrm{~m}$ telescope, to which a uvby - $\beta$ spectrophotometer was attached, was utilized at all times. Table 1 lists the log of the observations.

\subsection{Data Acquisition and Reduction}

A well-defined and calibrated $u v b y-\beta$ data set provides an efficient way to investigate the pulsational nature of the stars as well as the physical conditions, such as effective temperature and surface gravity, by comparing the unreddened indexes obtained with the theoretical models. The calibrations for unreddening have already been described and used in previous analyses (Peña \& Peniche; 1994; Peña \& Sareyan, 2006).

As was stated in Peña et al. (2016) reporting on BO Lyn, the observational pattern, as well as the reduction procedure, have been employed at the SPM Observatory since 1986 and hence, have been described repeatedly. A detailed description of the methodology can be found in Peña et al. (2007). 
TABLE 1

LOG OF THE OBSERVATIONS

\begin{tabular}{ccccc}
\hline $\begin{array}{c}\text { Date } \\
\text { YYY/MM/DD }\end{array}$ & Target 1 & Target 2 & Target 3 & $\begin{array}{c}\text { HJD } \\
\text { - 2450000(day) }\end{array}$ \\
\hline $2013 / 06 / 23$ & HD115520 & NGC 6913 & RRs & 7687 \\
$2013 / 06 / 24$ & HD15520 & NGC 6913 & & 7688 \\
$2013 / 06 / 25$ & HD115520 & NGC 6913 & & 7689 \\
$2013 / 06 / 26$ & HD115520 & & RRs & 7690 \\
$2018 / 10 / 28$ & HD220735 & GP And & & 8419 \\
$2018 / 10 / 31$ & HD220735 & & Cephs & 8422 \\
$2018 / 11 / 01$ & HD220735 & GP And & & 8423 \\
$2018 / 11 / 02$ & & GP And & Cephs & 8424 \\
$2018 / 11 / 03$ & & Cephs & 8425 \\
$2018 / 11 / 04$ & HD26738 Tau & & & 8426 \\
\hline
\end{tabular}

TABLE 2

TRANSFORMATION COEFFICIENTS OBTAINED FOR THE OBSERVED SEASONS

\begin{tabular}{cccccccc}
\hline Season & B & D & F & J & H & I & L \\
\hline June 2013 & -0.016 & 0.974 & 1.028 & 0.009 & 1.049 & 0.162 & -1.345 \\
$\sigma$ & 0.052 & 0.018 & 0.040 & 0.022 & 0.034 & 0.030 & 0.054 \\
\hline Oct 2018 & 0.021 & 0.989 & 1.028 & -0.017 & 1.019 & 0.180 & -1.398 \\
$\sigma$ & 0.062 & 0.007 & 0.026 & 0.017 & 0.020 & 0.032 & 0.034 \\
\hline
\end{tabular}

The observations were done over two different seasons. HD 115520 was observed in 2013, mostly in uvby without $\mathrm{H} \beta$ measurements, whereas the other two stars, HD 220735 and HD 26738, were observed in 2018 in uvby - $\beta$. Nevertheless, in both seasons the procedure was the same: at least five ten-second integrations of each star and one ten-second integration of the sky for the uvby filters as in the narrow and wide filters that define $\mathrm{H} \beta$ were taken for each measurement. The time difference between the two sets is $0.0006 \mathrm{~d}$. What must be emphasized here are the transformation coefficients for the observed seasons (Table 2) and the season errors, which were evaluated using the observed standard stars.

The best way to determine the accuracy of our observations is through the differences in magnitude and color indexes $\left(\mathrm{V}, b-y, m_{1}, c_{1}\right.$ and $\left.\mathrm{H} \beta\right)$ of the standard stars. In the 2013 season, 373 data points of sixteen standard stars were measured. The corresponding standard deviations of the mean values for $\left(\mathrm{V}, b-y, m_{1}, c_{1}\right)$ were $(0.019,0.006,0.007$ and $0.014)$. In the 2018 season thirty-four standard stars were measured for a total of ninety-four points in uvby and 68 points in $\mathrm{H} \beta$, respectively; the calcu- lated uncertainties were $(0.054,0.012,0.019,0.025$, 0.012 ), which provide a numerical evaluation of our uncertainties. Emphasis is made on the large range of the standard stars in the magnitude and color indexes values in both seasons: $V:(5.62,8.00) ;(b-y)$ : $(-0.09,0.88) ; m_{1}:(-0.09,0.67) ; c_{1}:(-0.02,1.32)$ and $\mathrm{H} \beta:(2.50,2.90)$ for the 2018 season. Similar ranges were obtained in the 2013 season.

To verify the consistency of the data from our derived standard star values, mean values for each one, as well as their standard deviations were calculated. For the 2018 season these are presented in Table 3 by decreasing brightness. The last column of this table is $\mathrm{N}$, the number of entries.

Table 4 lists the photometric values obtained of HD 26738. In this table Column 1 reports the time of the observation in HJD, Columns 2 to 5 list the Strömgren values $\mathrm{V},(b-y), m_{1}$ and $c_{1}$, respectively; Column $6, \mathrm{H} \beta$. The photometry of the other two stars has been sent to the Variable Star Index (VSX) for publication, and is also available upon request. The photometry of the light curves of the variables in the $\mathrm{V}$ filter is presented in Figures 1, 2 and 3. 
TABLE 3

MEAN PHOTOMETRIC VALUES AND STANDARD DEVIATIONS OF THE STANDARD STARS OF THE 2018 SEASON

\begin{tabular}{|c|c|c|c|c|c|c|c|c|c|c|c|}
\hline ID & $\mathrm{V}$ & $(b-y)$ & $m_{1}$ & $c_{1}$ & $\beta$ & $\sigma \mathrm{V}$ & $\sigma(b-y)$ & $\sigma m_{1}$ & $\sigma c_{1}$ & $\sigma \beta$ & $\mathrm{N}$ \\
\hline HR8085 & 5.195 & 0.669 & 0.650 & 0.167 & 2.529 & 0.054 & 0.014 & 0.033 & 0.020 & 0.018 & 6 \\
\hline HD013871 & 5.763 & 0.275 & 0.180 & 0.513 & 2.664 & 0.052 & 0.009 & 0.021 & 0.008 & 0.014 & 4 \\
\hline HD057006 & 5.887 & 0.354 & 0.111 & 0.525 & & & & & & & 1 \\
\hline HD035520 & 5.901 & 0.138 & 0.071 & 1.331 & 2.720 & 0.014 & 0.003 & 0.004 & 0.003 & 0.024 & 4 \\
\hline HD015335 & 5.917 & 0.367 & 0.167 & 0.386 & 2.594 & 0.076 & 0.004 & 0.005 & 0.006 & 0.019 & 4 \\
\hline HD224165 & 5.965 & 0.718 & 0.539 & 0.236 & & & & & & & 1 \\
\hline HD033203 & 5.996 & 0.619 & -0.185 & 0.005 & 2.597 & 0.024 & 0.008 & 0.016 & 0.012 & 0.025 & 5 \\
\hline HR8086 & 6.053 & 0.811 & 0.636 & 0.102 & 2.513 & 0.104 & 0.006 & 0.014 & 0.009 & 0.016 & 6 \\
\hline HD202314 & 6.201 & 0.689 & 0.451 & 0.297 & 2.618 & 0.111 & 0.006 & 0.011 & 0.007 & 0.020 & 6 \\
\hline HD015596 & 6.219 & 0.560 & 0.273 & 0.392 & 2.547 & 0.051 & 0.002 & 0.003 & 0.005 & 0.010 & 4 \\
\hline HD221661 & 6.224 & 0.602 & 0.414 & 0.353 & & & & & & & 1 \\
\hline HD033632 & 6.480 & 0.340 & 0.146 & 0.353 & 2.647 & 0.033 & 0.006 & 0.011 & 0.009 & 0.027 & 5 \\
\hline HD028354 & 6.534 & 0.008 & 0.115 & 0.793 & 2.809 & 0.020 & 0.010 & 0.017 & 0.011 & 0.011 & 4 \\
\hline HR8389 & 6.550 & 0.032 & 0.115 & 1.122 & 2.818 & 0.014 & 0.007 & 0.012 & 0.005 & 0.004 & 6 \\
\hline HD013936 & 6.583 & 0.019 & 0.141 & 1.128 & 2.798 & 0.090 & 0.006 & 0.008 & 0.009 & 0.015 & 4 \\
\hline HD043461 & 6.617 & 0.015 & 0.063 & 0.583 & 2.668 & 0.042 & 0.009 & 0.016 & 0.012 & 0.027 & 4 \\
\hline HD042089 & 6.626 & 0.582 & 0.335 & 0.535 & 2.627 & 0.037 & 0.004 & 0.010 & 0.011 & 0.004 & 4 \\
\hline HD012884 & 6.764 & 0.087 & 0.215 & 0.899 & 2.866 & 0.076 & 0.003 & 0.006 & 0.009 & 0.029 & 4 \\
\hline HD018066 & 6.956 & 0.755 & 0.557 & 0.328 & 2.560 & 0.055 & 0.004 & 0.004 & 0.008 & 0.018 & 4 \\
\hline HD044812 & 6.998 & 0.667 & 0.455 & 0.300 & 2.589 & 0.033 & 0.004 & 0.004 & 0.004 & 0.029 & 4 \\
\hline HD055036 & 7.000 & 0.268 & 0.014 & 1.308 & & & & & & & 1 \\
\hline HD208344 & 7.199 & 0.071 & 0.184 & 1.097 & 2.890 & 0.037 & 0.005 & 0.009 & 0.008 & 0.008 & 6 \\
\hline HD049564 & 7.371 & 0.836 & 0.708 & 0.356 & & & & & & & 1 \\
\hline HD204132 & 7.542 & 0.368 & 0.068 & 1.327 & 2.755 & 0.034 & 0.003 & 0.005 & 0.010 & 0.024 & 6 \\
\hline HD028304 & 7.723 & 0.147 & 0.031 & 0.614 & 2.730 & 0.036 & 0.004 & 0.003 & 0.003 & 0.013 & 4 \\
\hline HD013801 & 7.885 & 0.199 & 0.179 & 0.688 & 2.740 & 0.030 & 0.017 & 0.019 & 0.008 & 0.021 & 4 \\
\hline HD031125 & 7.955 & 0.030 & 0.176 & 1.001 & 2.931 & 0.071 & 0.003 & 0.004 & 0.010 & 0.030 & 4 \\
\hline HD219364 & 7.958 & 0.679 & 0.541 & 0.376 & 2.593 & 0.026 & 0.024 & 0.023 & 0.009 & 0.021 & \\
\hline HD047777 & 7.985 & -0.052 & 0.085 & 0.084 & 2.649 & 0.066 & 0.004 & 0.009 & 0.022 & 0.017 & 3 \\
\hline HD013997 & 8.010 & 0.464 & 0.331 & 0.367 & 2.577 & 0.055 & 0.023 & 0.025 & 0.006 & 0.019 & 4 \\
\hline HD207608 & 8.069 & 0.313 & 0.146 & 0.533 & 2.669 & 0.110 & 0.005 & 0.004 & 0.004 & 0.014 & 6 \\
\hline HD048691 & 8.212 & 0.144 & -0.032 & -0.013 & & & & & & & 2 \\
\hline
\end{tabular}

\section{PERIODIC CONTENT}

For the two previously known variable stars, HD 115520 and HD 220735, we have significantly extended the time basis. Hence, period analysis of both should give more significant results. The numerical package of Period04 (Lenz \& Breger, 2005) was used.

Searching for new short period variables, HD 26738 was found to be a new variable. This star, according to SIMBAD (Wenger et al. 2000), has no previous reports on its variability.

A brief description for each star is presented.

\subsection{HD 115520}

This star was first determined to be variable while it was being used as a standard star from a season in 2005. The photometric data of this star presented a large scatter in comparison with the other standard 
TABLE 4

$u v b y-\beta$ PHOTOELECTRIC PHOTOMETRY OF HD 26738

\begin{tabular}{cccccc}
\hline HJD & $\mathrm{V}$ & $(b-y)$ & $m_{1}$ & $c_{1}$ & $\beta$ \\
\hline 425.8569 & 9.472 & & & 0.932 & \\
425.9039 & 9.517 & 0.250 & 0.165 & 0.925 & \\
425.8593 & 9.465 & 0.247 & 0.171 & & \\
425.8965 & 9.510 & 0.253 & 0.164 & 0.921 & 2.885 \\
425.8489 & 9.497 & 0.251 & 0.164 & 0.919 & 2.884 \\
425.8674 & 9.447 & 0.243 & 0.173 & & \\
425.8745 & 9.462 & 0.244 & 0.165 & 0.929 & \\
425.8464 & 9.501 & & & 0.924 & 2.879 \\
425.8649 & 9.454 & 0.244 & 0.163 & 0.939 & \\
425.9150 & 9.465 & 0.246 & & 0.933 & 2.873 \\
425.8386 & 9.504 & 0.257 & 0.158 & 0.920 & 2.872 \\
425.8411 & 9.509 & 0.256 & 0.158 & 0.929 & 2.870 \\
425.8309 & 9.490 & 0.257 & 0.156 & 0.925 & 2.867 \\
425.8209 & 9.459 & 0.247 & 0.164 & 0.927 & 2.864 \\
425.8773 & 9.468 & 0.249 & 0.161 & 0.932 & 2.864 \\
425.9069 & & 0.247 & 0.170 & 0.912 & \\
425.9179 & 9.469 & & 0.166 & 0.935 & 2.861 \\
425.8235 & 9.470 & 0.248 & 0.162 & 0.932 & 2.854 \\
425.8333 & 9.495 & 0.256 & 0.156 & 0.926 & \\
\hline
\end{tabular}

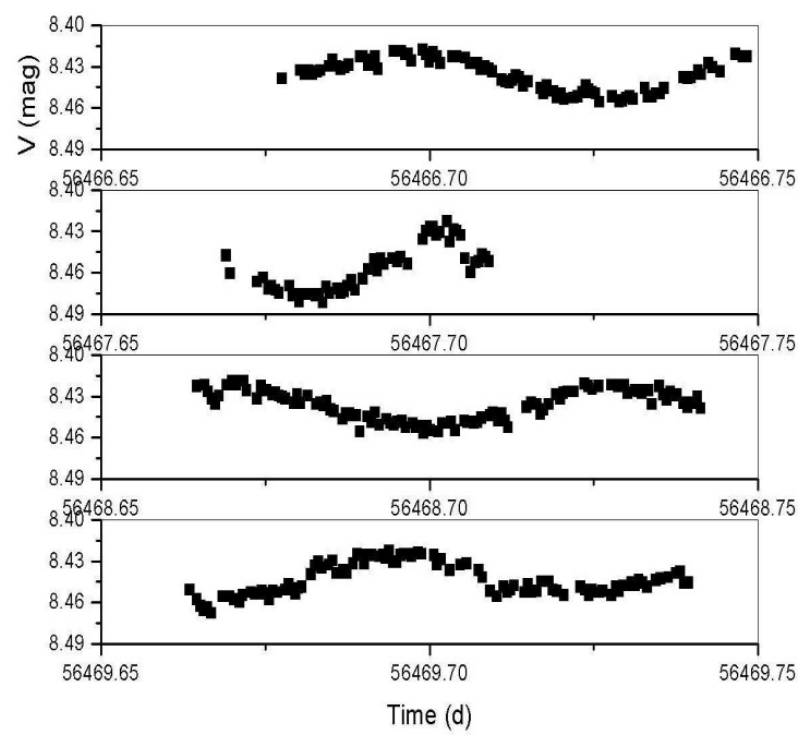

Fig. 1. Light curves of HD115520 in the V filter obtained in $u v b y-\beta$ absolute photometry. Each night is shown in descending order . To obtain the adequate HJD, 2400000 must be added.

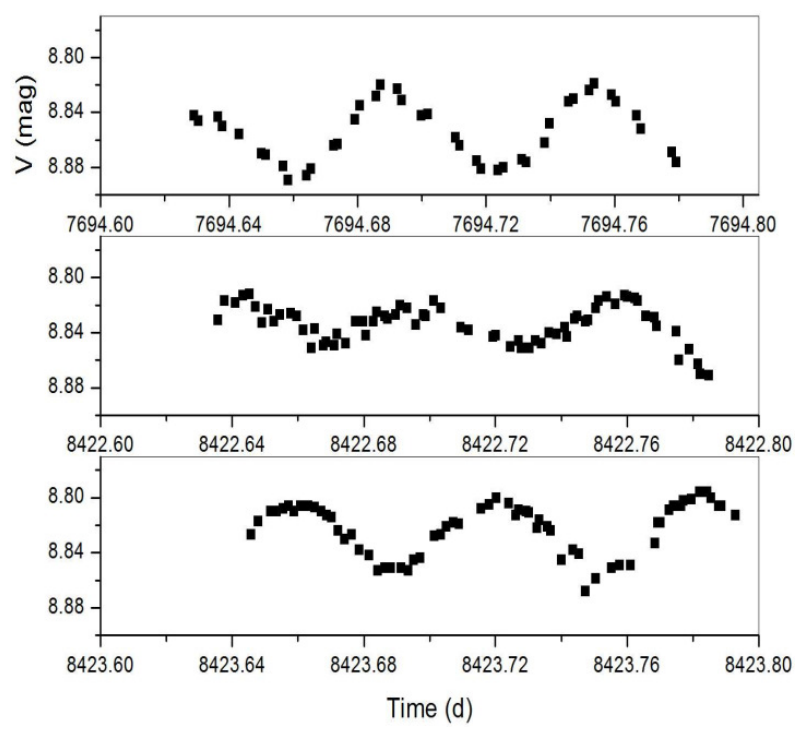

Fig. 2. Light curves of HD 220735 in the V filter obtained in $u v b y-\beta$ absolute photometry. At the top, the night of the previous season. To convert time shown into HJD, 2450000.00 must be added.

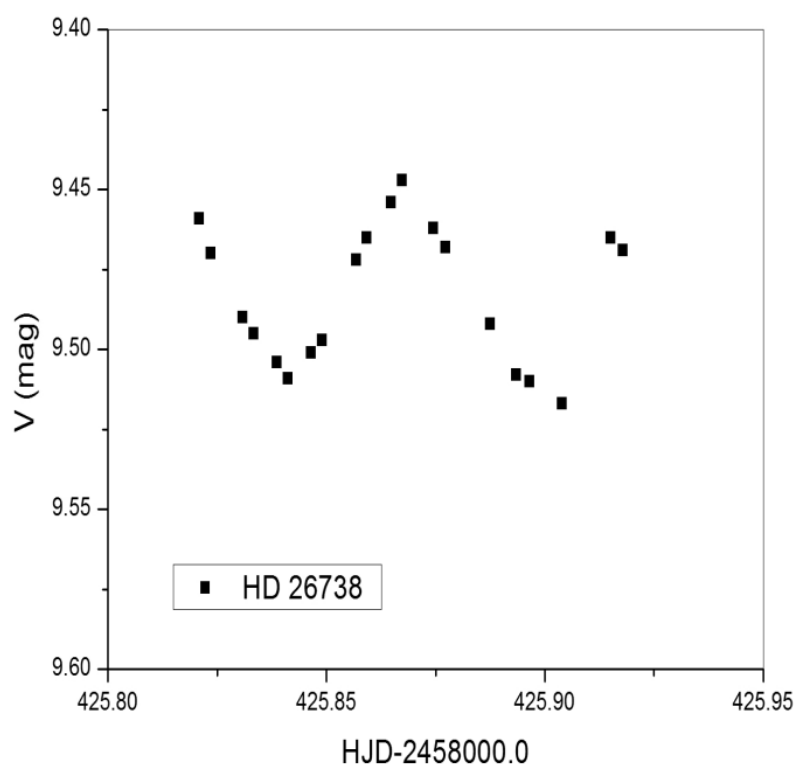

Fig. 3. Light curve of HD 26738 in the $\mathrm{V}$ magnitude of the $u v b y-\beta$ photoelectric photometry. To convert time shown into HJD, 2450000.00 must be added. 
TABLE 5

OUTPUT OF PERIOD04 CALCULATED WITH THE V MAGNITUDE OF THE STAR HD115520 WITH SEVERAL DATA SETS

\begin{tabular}{cllll}
\hline Considered data & Nr. & Frequency & Amplitude & Phase \\
\hline Set 1 & F1 & 18.00 & 0.0130 & 0.2789 \\
& F2 & 16.16 & 0.01442 & 0.6888 \\
\hline Set 2 & F1 & 17.8375 & 0.0131 & 0.1028 \\
& F2 & 14.7537 & 0.0108 & 0.2612 \\
& F3 & 16.51521 & 0.0070 & 0.5646 \\
\hline Set 3 & F1 & 17.6933 & 0.01503 & 0.5483 \\
& F4 & 15.9967 & 0.00473 & 0.8836 \\
& F6 & 14.5667 & 0.0063 & 0.0400 \\
\hline
\end{tabular}

Notes: Set 1: data from 2005 and 2006 (Fox-Machado et al (2007). Set 2: adds V magnitude of the uvby data (Peña et al. 2007). Set 3: includes the 2013 data (present paper).

stars used and this dispersion led the authors to suspect the variable nature of the star. New observations were performed in 2006 to verify its variability (Fox Machado et al. 2007) and it was observed again in 2006 to verify its variability (Fox Machado et al. 2007). It was re-observed in 2007 and the analysis published (Peña et al, 2007). Finally it was observed again in 2013. The time basis is, therefore, ten nights in 2600 days. The analysis of the shorter data groups has already been done and reported. Here we merely copy the published results in Table 5 . At the bottom we add the analysis obtained when analysing the entire data, but we must keep in mind that the gap between observations is huge, six years. Figure 4 presents the periodograms of HD 115520 with the San Pedro Martir Observatory's V data.

\section{2. $H D 220735$}

Peña et al. (2019) reported from observations taken in 2016 that the periodic behavior of the star was determined from one night that covered a sufficient time span to cover two cycles. The difference of these maxima gave $0.0658 \mathrm{~d}$, which gives a coarse period of pulsation of this star.

The other technique which was used for this star in the present work is time series analysis. These data gave the results listed in Table 6 with a zero point of $5.39 \mathrm{mag}$, residuals of $0.0049 \mathrm{mag}$ and 5 iterations. This frequency coarsely agrees with that determined by the difference of the two maxima: $0.0658 \mathrm{~d}$. The analysis of Period04 is presented in Figure 2. Beginning at the top is the periodogram of the original data; next are the consecutive sets of residuals. The scale of the $\mathrm{Y}$ axis shows the relative
TABLE 6

OUTPUT OF PERIOD04 WITH THE V MAGNITUDE OF HD 220735 OF THE $u v b y-\beta^{*}$

\begin{tabular}{cccc}
\hline Nr. & Frequency & Amplitude & Phase \\
\hline F1 & 16.5974 & 0.0200 & 0.8674 \\
F3 & 13.2807 & 0.0088 & 0.2043 \\
F3 & 27.6206 & 0.0033 & 0.8021 \\
\hline
\end{tabular}

* Data from 2016 and present paper.

importance of the residuals. However, it is obvious that the data of only one night cannot provide an accurate period determination. To complicate things more, this preliminary analysis suggests the presence of a second frequency, a common phenomenon among $\delta$ Scuti stars.

The star was observed again in December, 2018 for two nights. Combining the data the entire time basis is 732 days. Figure 5 presents the periodograms of HD 220735 with the SPM V data.

\section{3. $H D 26738$}

The star was observed on the night of Nov 3, 2018 (JD 2458424 ) for a short period of time (three hours). Although its variability is evident (Figure 3) the series analysis with Period04 gave the following results despite the shortness of the observing time span. A period of $0.15 \mathrm{~d}$ is determined from the consecutive minima whereas Period04 gave a frequency of $16.955 \mathrm{c} / \mathrm{d}$ with an amplitude of 0.0312 and a phase of 0.98. Prewhitening this frequency, a sec- 


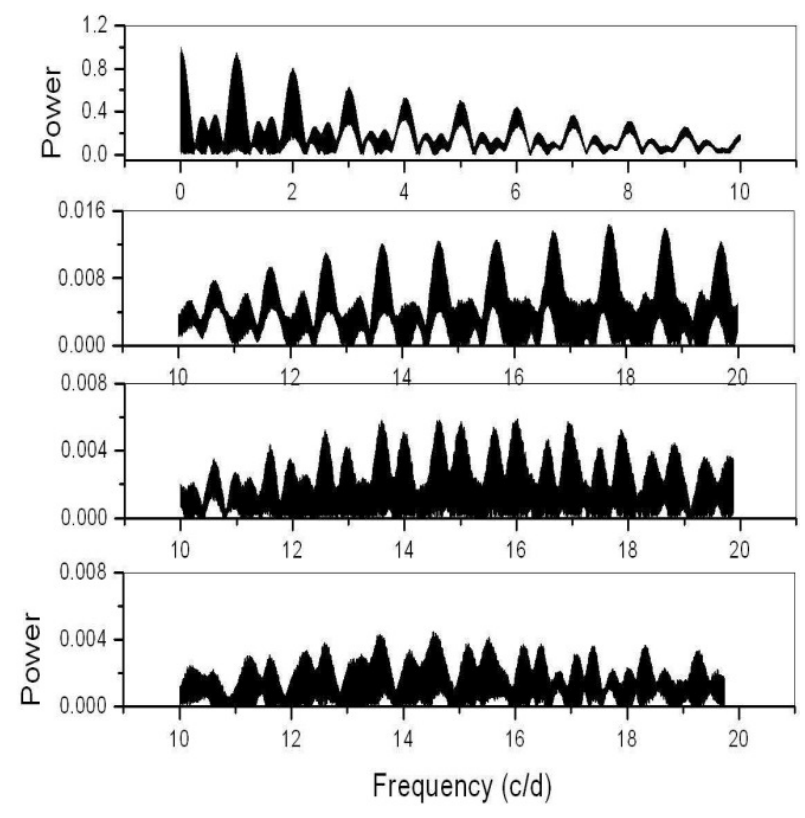

Fig. 4. Periodograms of HD 115520 with the SPM V data. At the top is the periodogram of the window; descending, the periodogram of the original data and subsequently, the periodogram of the residuals after subtracting each frequency. We call attention to the scale of the $\mathrm{Y}$ axis to show the relative importance of each frequency. Although the periodogram was evaluated from 0 to $50 \mathrm{c} / \mathrm{d}$ we merely present a limited frequency range, where the pulsational frequencies were found.

ond peak at $9.147 \mathrm{c} / \mathrm{d}$ with an amplitude of 0.0057 and a phase of 0.568 was obtained. The residuals were 0.00388 mag.

\subsection{Discussion}

Since their discovery neither HD 115520 nor HD 220735 had been studied for period determination. After the first report, more information was gathered, but no period analysis was done. In the present paper, two different approaches were utilized to determine the period of the pulsation.

In the first one, differences in consecutive times of maximum light were evaluated to determine a coarse period; the second method utilized was a time series analyses. The set employed was that of the $\mathrm{V}$ magnitude of the $u v b y-\beta$ photoelectric analysis in the present paper.

\section{PHYSICAL PARAMETERS}

\subsection{Spectral Type Determination}

To determine physical parameters, unreddened photometric values have to be determined through ap-

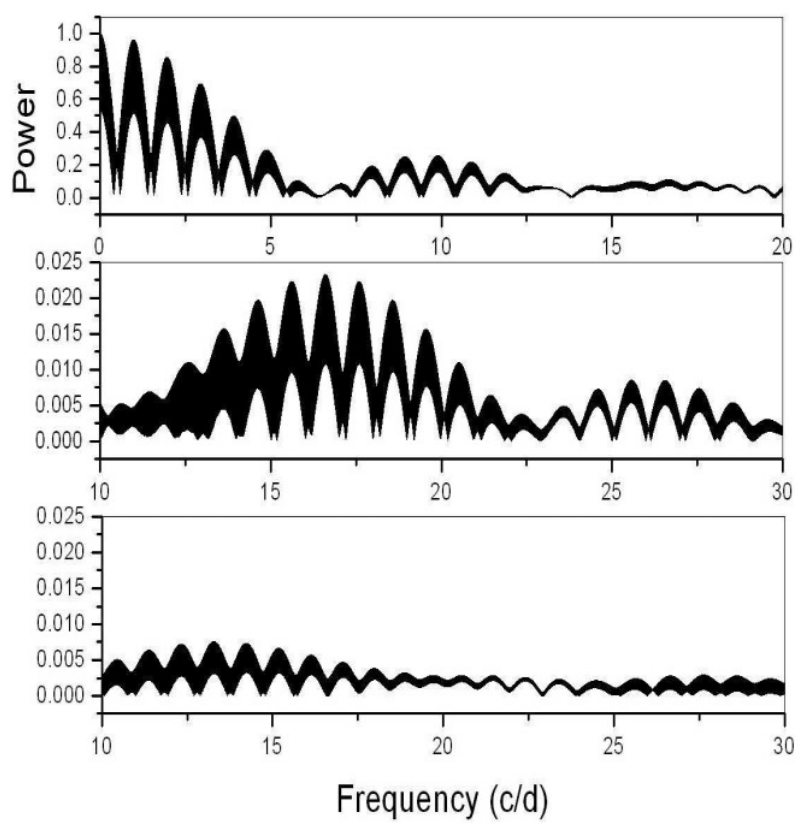

Fig. 5. Periodograms of HD 220735 with the SPM V data. Top to bottom: first is the periodogram of the window function. Middle, the output that provides the first frequency $(16.597 \mathrm{c} / \mathrm{d})$, and bottom, the result of the set of residuals subtracting this frequency at $13.280 \mathrm{c} / \mathrm{d}$ ). We call attention to the scale of the $\mathrm{Y}$ axis to show the relative importance of each frequency.

propriate calibrations. These calibrations were proposed by Nissen (1988) for A and F type stars. Therefore, it is necessary to first determine if the range of variation in spectral class of the involved stars lies in these limits. The spectral types can be determined very accurately with the $u v b y-\beta$ photometric data. We determined their unreddened photometric indexes $\left[m_{1}\right]$ and $\left[c_{1}\right]$ and positioned them in the analogous plot determined for the open cluster Alpha Per, whose stars have well-determined spectral types and whose membership has been established.

\subsection{HD115520}

The best way to determine the accuracy of our observations is through the differences in magnitude and colors ( $\mathrm{V}, b-y, m_{1}, c_{1}$ and $\mathrm{H} \beta$ ) of the standard stars, where $c_{1}$ and $m_{1}$ are parameters defined by the well-known color indexes: $m_{1}=(v-b)-(b-y)$ and $c_{1}=(u-v)-(v-b)$. For HD 115520 Fox Machado et al. (2007) stated that its position in the $\left[m_{1}\right]-\left[c_{1}\right]$ diagram establishes it to be an $\mathrm{A} 8 \mathrm{~V}$ star, a classification that was corroborated in Peña et al. (2007). 


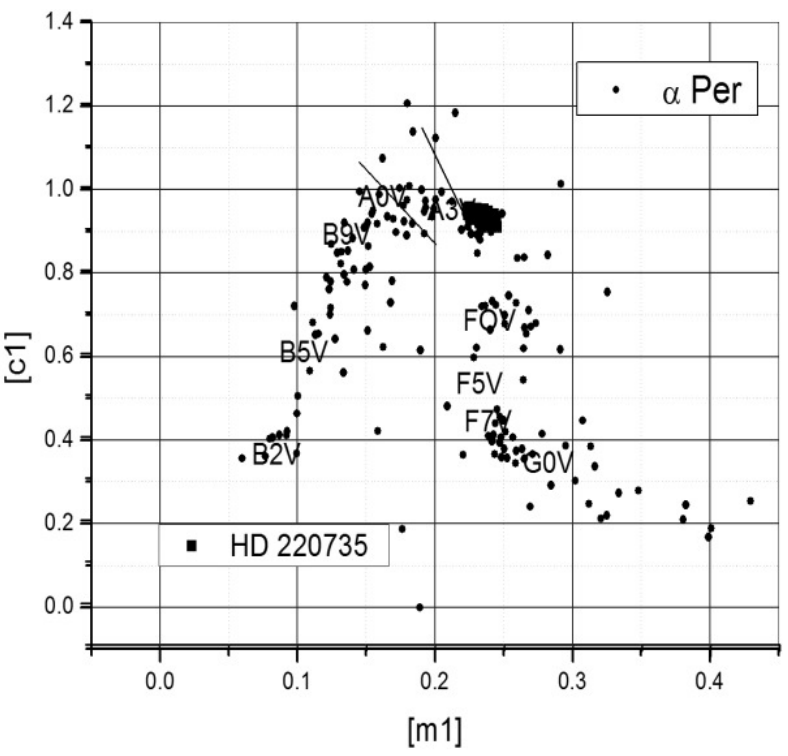

Fig. 6. Position of the HD 220735 star in the $\left[m_{1}\right]-\left[c_{1}\right]$ diagram of the open cluster Alpha Per (Peña \& Sareyan, 2006).

\subsection{HD 220735}

The analysis of HD 220735 reported by Peña et al. (2019) stated that the spectral type of this star is A3-A4. This determination was done by locating the unreddened color indexes of the uvby $-\beta$ photometry in the $\left[m_{1}\right]-\left[c_{1}\right]$ diagram of this star compared to those determined for the open cluster Alpha Per (Peña et al., 2006) for which there is accurate determination of the spectral types for its stars and its photometry. This is shown schematically in Figure 6.

\section{4. $H D 26738$}

Figure 7 shows the position of this star in the $\left[m_{1}\right]-$ $\left[c_{1}\right]$ diagram. Its spectral type corresponds to an $\mathrm{A}$ main sequence star around A4 or A5.

\subsection{Physical Parameters Determination}

In view of the fact that the three stars have spectral classes typical of $\delta$ Scuti stars, A8 for HD 115520, A3-A4 for HD 220735 and A4-A5 for HD 26738, we can apply the prescription of Nissen (1988) for the three to determine their reddening. This procedure has been explained elsewhere (see for example Peña et al. (2007)).

To determine the range of the effective temperature and surface gravity in which the stars vary,

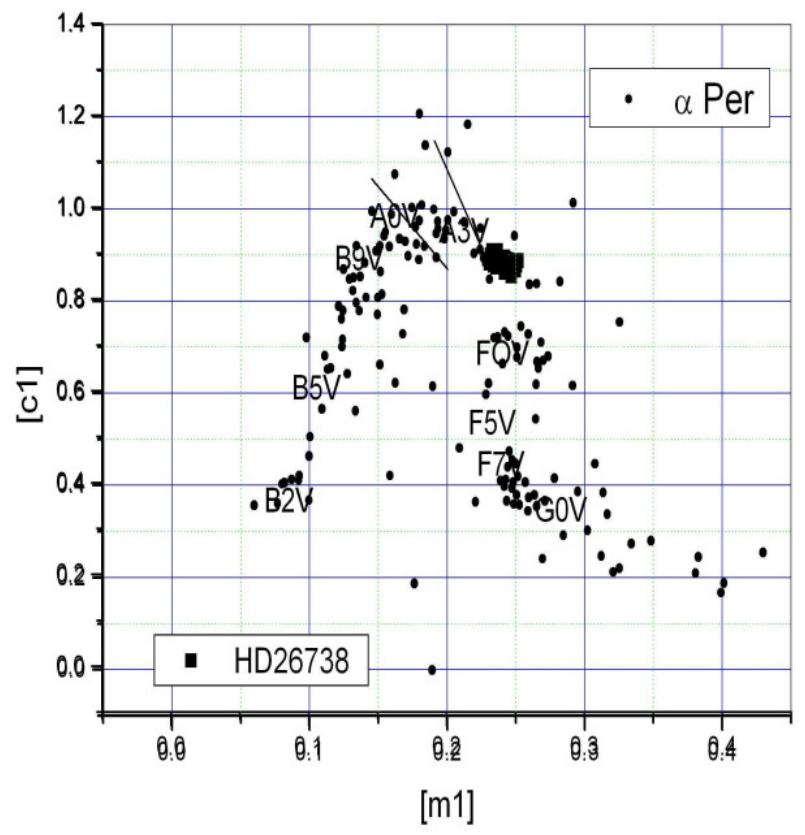

Fig. 7. Position of the HD 26738 star in the $\left[m_{1}\right]-\left[c_{1}\right]$ diagram of the open cluster Alpha Per (Peña \& Sareyan, 2006).

we must locate the determined unreddened points in some theoretical grids such as those of Lester, Gray and Kurucz (1986, hereinafter LGK86) developed for $u v b y-\beta$ photometric data for several metallicities. However, before locating the unreddened points in the theoretical grids, a metallicity for each star has to be assumed. For each star its unreddened points $(b-y)$, and $m_{1}$ were plotted in a diagram of the LGK86 grids built for $\log \mathrm{g}$ of 4 and for different metallicities. The position in the $(b-y)$ vs. $m_{1}$ diagram of the unreddened points indicates the metallicity $[\mathrm{Fe} / \mathrm{H}]$ of each star. They were: for HD 115520 a metallicity of 0.0 ; for HD 26738, of 1.0 and for HD 220735 values spread out between 0.5 and 1.0. These are the metallicities that were assumed.

As can be seen in Figure 8, for the case of $[\mathrm{Fe} / \mathrm{H}]=1.0$, the HD 220735 star varies between an effective temperature of $7500 \mathrm{~K}$ and $8200 \mathrm{~K}$; the surface gravity $\log g$ lies around 4.0. The star HD 26738 (Figure 9), on the other hand, varies between 7500 and $8100 \mathrm{~K}$ with a $\log \mathrm{g}$ slightly larger than 4.0. In the case of HD 115520 since no new $H \beta$ observations were done in 2013 we rely on the 2007 findings for unreddening and obtained: Te around $7700 \mathrm{~K}$ and $\log g$ close to 4.0 . 
TABLE 7

DETERMINED PARAMETERS FOR THE VARIABLE STARS

\begin{tabular}{lccc}
\hline \multicolumn{1}{c}{ Parameter } & HD 115520 & HD 220735 & HD 26738 \\
\hline Reddening [mag]E(b-y) & 0.000 & $0.051 \pm 0.016$ & $0.173 \pm 0.011$ \\
Distance [pc] & $119 \pm 7$ & $280 \pm 35$ & $205 \pm 15$ \\
Distance modulus [mag] & $5.4 \pm 0.1$ & $7.2 \pm 0.3$ & $6.6 \pm 0.2$ \\
{$[\mathrm{Fe} / \mathrm{H}]$} & 0.0 & 1.0 & 1.0 \\
\hline
\end{tabular}

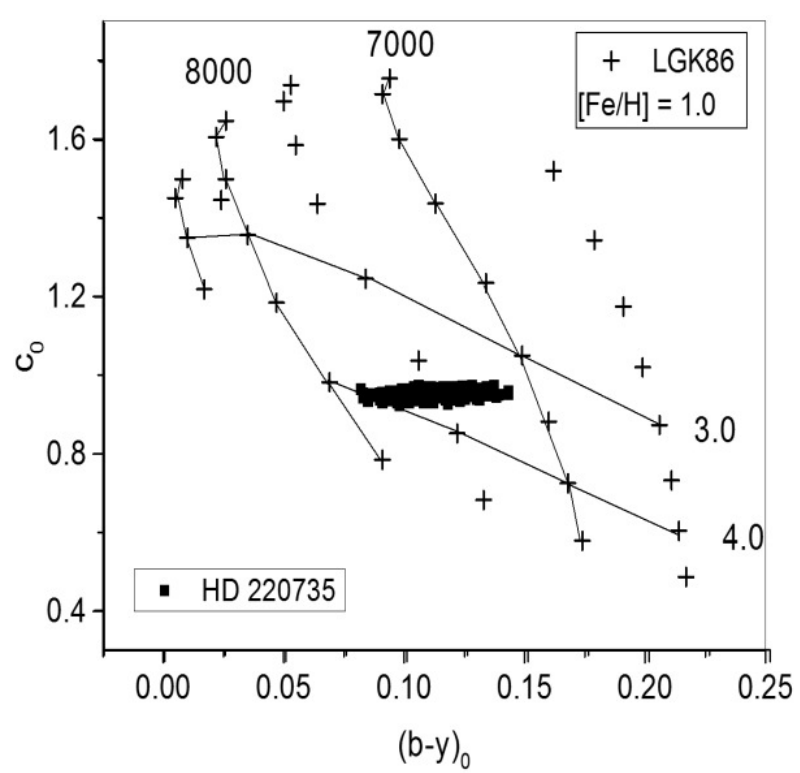

Fig. 8. Position of the HD 220735 star in the grids of LGK86 for a metallicity of 1.0.

\subsection{Physical Parameters Conclusions}

New observations in uvby $-\beta$ photoelectric photometry were carried out on the three variable stars.

Reddening, as well as the unreddened indexes, were determined from Nissen's (1988) calibrations. These served to determine the physical characteristics of the HD 220735 star: effective temperature in a range from $7000 \mathrm{~K}$ to $7700 \mathrm{~K}$ and $\log g$ from 3.6 to 4.3 from two methods: (1) from the location of the unreddened indexes in the LGK86 grids and (2) through the theoretical relation (P\&J72).

The application of the above mentioned numerical unreddening package of Nissen's (1988) provided the results for HD 115520 and HD 226738. The results are summarized in Table 7 which lists the reddening $E(b-y)$, distance modulus, DM and distance (in pc) as well as the determined metallicity.

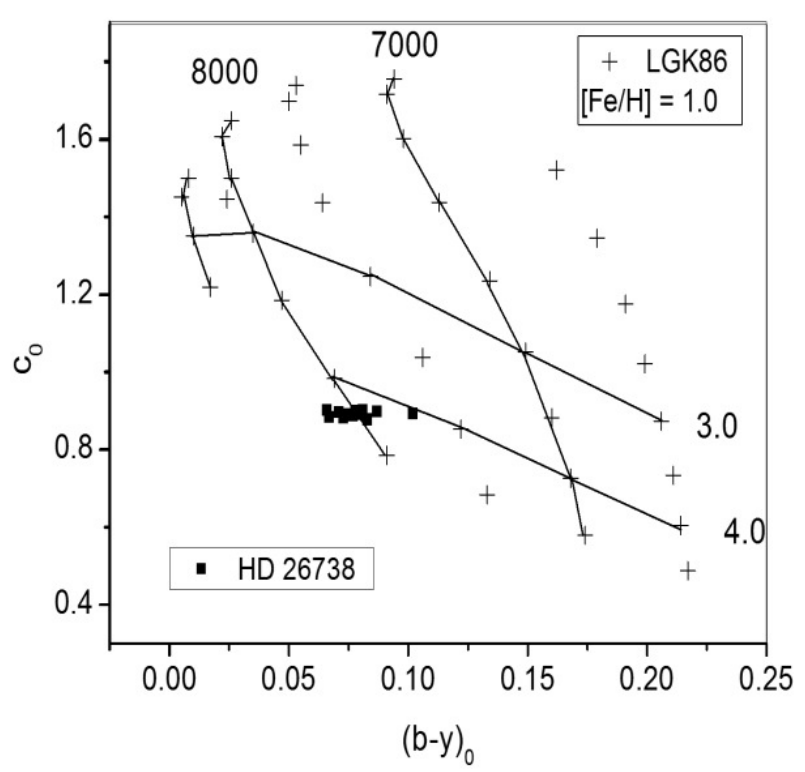

Fig. 9. Position of the HD26738 star in the grids of LGK86 for a metallicity of 1.0

\section{CONCLUSIONS}

In this study we present our findings on HD 115520 and HD 220735 which were previously reported to be variables. We confirmed their spectral types from $u v b y-\beta$ photoelectric photometry. We did the same for the unreported variable star HD 22738. The extended time basis of the observed stars served to determine their period of pulsation more accurately. Physical characteristics were calculated by determining first their reddening and plotting the unreddened indexes in the theoretical grids of LGK86. The determined values are consistent with their spectral types.

We would like to thank A. Pani, and the staff of the OAN at SPM for their assistance in securing the observations and the GAOOT group (J. Guillen and D. Segura) for fruitful discussions. This work was partially supported by PAPIIT IN104917, IG100620 and PAPIME PE113016. Proofreading and typing were done by J. Miller and J. Orta, respectively. 
C. Villarreal, C. Guzmán, F. Ruiz, A. Díaz and G. Pérez assisted us at different stages. AAS, SB, HH, ARL, ALZ and AZ thank the IA for allotting the telescope time. The comments and suggestion of an anonymous referee improved this article. We have made use of the SIMBAD databases operated at CDS, Strasbourg, France and NASA ADS Astronomy Query Form.

\section{REFERENCES}

Fox-Machado, L., Peña, J. H., Muñoz, G., \& Vargas, B. 2007, CoAst, 151, 26

Lenz, P. \& Breger, M. 2005, CoAst, 146, 53
Lester, J. B., Gray, R. O., \& Kurucz, R. L. 1986, ApJS, 61,509

Nissen, P. 1988, A\&A, 199, 146

Peña, J. H. \& Peniche, R. 1994, RMxAA, 28, 139

Peña, J. H. \& Sareyan, J.-P. 2006, RMxAA, 42, 179

Peña, J. H., Sareyan, P., Cervantes-Sodi, B., et al. 2007, RMxAA, 43, 217

Peña, J. H., Villarreal, C., Piña, D. S., et al. 2016, RMxAA, 52, 385

Peña, J. H., Soni, A., Rentería, A., \& Piña, D. 2019, IBVS, 6260, 1

Petersen, J. O. \& Jørgensen, H. E. 1972, A\&A, 17, 367

Wenger, M., Ochsenbein, F., Egret, D., et al. 2000, A\&AS, 143, 9

H. Huepa, J. H. Peña, and A. Rentería: Observatorio Astronómico Nacional de Tonantzintla, México.

S. B. Juárez, J. H. Peña, A. Rentería, A. A. Soni, and A. L. Zuñiga: Facultad de Ciencias, Universidad Nacional Autónoma de México, México.

J. H. Peña, A. Rentería, and A. A. Soni: Instituto de Astronomía, Universidad Nacional Autónoma de México, Apdo. Postal 70-264, Cd. de México (jhpena@astro.unam.mx). 